\title{
A Process for Co-Designing Educational Technology Systems for Refugee Children
}

\author{
George Alain \\ Institute of Educational \\ Technology \\ The Open University \\ Milton Keynes, UK \\ george.alain@open.ac.uk
}

\author{
Tim Coughlan \\ Institute of Educational \\ Technology \\ The Open University \\ Milton Keynes, UK \\ tim.coughlan@open.ac.uk anne.adams@open.ac.uk
}

\author{
Helen Yanacopulos \\ Faculty of Arts \& \\ Social Sciences \\ The Open University \\ Milton Keynes, UK \\ h.yanacopulos@open.ac.uk
}

\begin{abstract}
There is a growing interest in the potential for technology to facilitate emergency education of refugee children. However, designing in this space requires knowledge of the displaced population and the contextual dynamics surrounding it. Design should therefore be informed by both existing research across relevant disciplines, and from the practical experience of those who are on the ground facing the problem in real life. This paper describes a process for designing appropriate technology for these settings. The process draws on literature from emergency education, student engagement and motivation, educational technology, and participatory design. We emphasise a thorough understanding of the problem definition, the nature of the emergency, and of socio-cultural aspects that can inform the design process. We describe how this process was implemented leading to the design of a digital learning space for children living in a refugee camp in Greece. This drew on involving different groups of participants such as social-workers, parents, and children.
\end{abstract}

Co-design; Participatory design; refugees; child-computer interaction; educational technology.

\section{INTRODUCTION}

According to the United Nations High Commissioner for Refugees (UNHCR), there are at least 65 million forcibly displaced people worldwide. Around 25 million of these people are refugees fleeing their homeland, mostly because of war and persecution. $51 \%$ of refugees are children, and even in the most positive estimates, only $50 \%$ of these children are attending any form of schooling (UNHCR, 2016a, 2016b). Where they exist, educational experiences range from informal activities at refugee camps, to formal schools in host countries. There are numerous challenges that make education problematic in these settings. The absence of any education, or serious challenges to the quality of education received by these millions of war-affected and displaced children may create catastrophic consequences in the future.

There is a growing interest in the potential for technology to facilitate emergency education for refugees, but designing in this space requires knowledge of the displaced population and the contextual dynamics surrounding it. Design should therefore be informed by both existing research across relevant disciplines, and from those who are on the ground facing these problems in their lives. Furthermore, the suitability of designs and the appropriate implementation of educational technology systems varies depending on the specifics of each situation. There can be very different needs and challenges found in diverse contexts, such as in refugee camps, or urban-based formal or informal learning educational projects. There is no one-size-fit-all solution that can be implemented in any refugee education context.

The goal of our work is therefore to develop and evaluate a reusable process that can be applied to the design and implementation of systems to support the education of war-affected displaced children. The process has been derived through reference to existing research across disciplines including refugee studies, emergency education, motivation and engagement in education, and participatory design. This process has been implemented and evaluated in two case studies with Syrian refugees in Greece. One case study at Ritsona refugee camp will be discussed in this paper. A further case study was implemented in a formal education setting at four Greek schools hosting Syrian refugee children. Through evaluation of these case studies and their outcomes, we are creating a reusable approach to guide the effective and empathetic use of technology in these settings.

\section{LITERATURE REVIEW}

\subsection{Emergency Education}

Research on education in emergency settings describes educational challenges in the context of disasters and conflicts. Both cases involve mass 
displacement challenges such as severe living conditions, lack of resources, and a lack of accessible health and education services. Since conflict emergencies are related to war and fighting among different groups, there are often further challenges related to issues such as politics, violence, trauma, discrimination, extremism, depression, racism, and separation (Kagawa, 2005).

Researching in such contexts is a delicate process, and thus, participatory approaches involving the target population and stakeholders are key to sustainable and applicable initiatives (Talbot and Muigai, 1998; Pigozzi, 1999; Sinclair, 2002; Kagawa, 2005). Tawil and Harley (2004) also stress that educational content for refugee children must be chosen or created with a full understanding of the conflict dynamics. This is to avoid triggering any sensitive topics that could cause alienation or reenforce any conflict related trauma. The participatory development of awareness of the experiences of the specific population is therefore central in our process.

Even where educational programs are in place for refugee children, a range of challenges can prevent children from benefiting. Disruptions caused by conflict or instability can mean that a great deal of schooling has been missed. This can create a loss of engagement and ambition. Issues related to the provision itself, such as language barriers, or low quality educational materials, can exacerbate psycho-social symptoms related to the severe lifestyle of many children, such as shame, hopelessness, and being exhausted and mentally withdrawn (Pigozzi, 1999; Betancourt, 2005; Dryden-Peterson, 2011). A lack of engagement, often expressed through inattention or dropping out of educational opportunities, is therefore a central theme in our process, and these identified challenges have parallels with research on educational engagement.

\subsection{Student Engagement}

Skinner and Pitzer (2012) discuss the dynamics of student engagement for coping and resilience. They provide a table of indicators of student engagement and student disaffection that reflect the same challenges discussed in emergency education literature. They suggest that teachers, peers, parents, the social context, and the nature of academic work can all support engagement. This informs the importance of involving different groups from the targeted location in the design process.

Outcomes of student engagement research have been integrated into a model based on selfdetermination theory (SDT). This is built on three pillars: Autonomy, Relatedness, and Competence (Niemiec and Ryan, 2009; Skinner and Pitzer, 2012). Furthermore, Spitzer (1996) provides a list of characteristics of motivating educational activities such as Action, Fun, Variety, Choice, Social Interaction, Error Tolerance, Measurement, Feedback, Challenge, and Recognition. The SDT concepts and Spitzer's motivational activities are employed in the process to guide the participatory design workshops and are also used to assess the educational content and ensure that it supports student engagement and motivation.

\subsection{Educational Technology Design}

Technology has been used extensively to support learning and student engagement and has yielded positive outcomes (Kadiyala Madhavi, 2000). Examples such as attractive visual multimedia, interactivity, or gamified learning, could hold the potential to engage refugee children with their education. However, a good match between learning objectives, teaching, and technology is essential. Particularly given the complex social and practical challenges of refugeehood, technology should not be seen as a solution, but as a possible mediator that tackle specific problems towards achieving specific identified goals.

The involvement of the target populations in devising these interventions is key, yet not trivial. Participatory Design (PD) literature offers guidance and analysis of means to involve end users and stakeholders from the early stages of system design. PD allows for designs that empower and involve these stakeholders to achieve applicable and sustainable systems (Scaife and Rogers, 1999; Muller and Druin, 2003).

Children are the target population of these design processes, and they can play a major role in PD. Druin (2002; 2003) suggests several roles which children can play such as users, testers, informant, and finally design partners which is the role that encapsulate all the other roles and is the one that children have played in our research. Scaife and Rogers (1999) specify that children are very good at telling us what motivates and engages them, and how they want things to be. In a valuable example of what is possible in this specific area, (Fisher, Yefimova and Yafi, 2016), ran participatory design workshops with refugee children to design technology solutions that can help them or the others around them. Our focus on education requires additional steps, and design workshops are preceded by a thorough problem definition stage to account for the educational needs and context.

\section{THE PROCESS}

The devised process has four stages. We start by understanding the conflict, population, and demography. This is followed by context and location orientation, then comes the problem 
definition and education conceptualization from the perspective of different participant groups, and then move to designing solutions that tackle the identified problems from previous stages. After the design process, we analyse the implementation and elicit feedback to evaluate the design process.

\subsection{Understanding the conflict and population}

This stage is derived from the literature on emergency education discussed in 2.1. The aim is to develop understanding of the conflict, the demography, and the possible contextual, and socio-cultural sensitive matters that may arise in the later stages which involve direct communication with the displaced population. This is essential to assess the educational content to avoid any clashes with the conflict and society related sensitive matters.

In the case study, the outcome of this stage was a clear understanding of the Syrian conflict, the tensions, population demography, with a list of possible sensitive topics that could affect the interaction with the researched community and educational content. The main researcher in our team is a native Arabic speaker who has lived in Syria, understands the culture, and had previously worked with Syrian refugees and refugee children. It is very important for the researchers and designers to understand the culture they will be working within.

\subsection{Location orientation}

This stage starts before and during arrival at the location. It aims to understand the location, the demography of the population living at the location, and the available resources. Moreover, this stage aims to identify the different groups that can participate in the problem definition and design stages, and to recognise their areas of expertise and the methods that can be used to get their input. Additionally, this stage aims to accomplish a positive relationship with the community and the participants as this facilitates the success of the later stages.

The case study took place at Ritsona refugee camp in Greece that hosts around 500 Syrian refugees. We identified three main groups of participants which are: on-site social-workers and NGO staff, parents, and children. Unlike the other case study, we were unable to work with educators as there was no educational provision in the camp.

To achieve a positive relationship, we used the servicing sampling method (Cohen, Manion and Morrison, 2013) which involved providing volunteer work to the community such as translation and activity planning for children and adults.

\subsection{Educational Problem Definition}

In this stage, we work on understanding the problem definition the perception of education from all different identified participant groups. This is to identify specific educational needs and challenges which technology can later be designed to tackle.

In the case study, we started with semi-structured interviews and focus groups with social-workers and NGO volunteers who provided us with a thorough understanding on the challenges faced by the community, the educational challenges, and their suggestions on the techniques to work with the refugees especially with children. We discussed current technology use in the camp and the possibilities for technology in education. We were provided with a list of available technologies at the camp. This is important as designs should later be scoped to the available and possible resources.

After gaining an understanding of how to work with residents, we conducted focus group meetings with refugee parents. We asked them about what they think of education, what their children think of education, and on what they think are the most critical educational needs that are currently not being met. Most parents prioritized literacy as most of the camp children are illiterate. Parents were appreciative of involving them in the discussions and this built trust which our early analysis see it as a key for successful research and designs. We asked for their consent to invite their children to our next activities which they approved and also proactively advised on the methods that engage their children.

The final stage in the problem definition was conducted with children. We asked them about their perception of education, the purpose of education, what they want to get educated about and why, and only after this we can start discussing the use of technology. We used methods considered

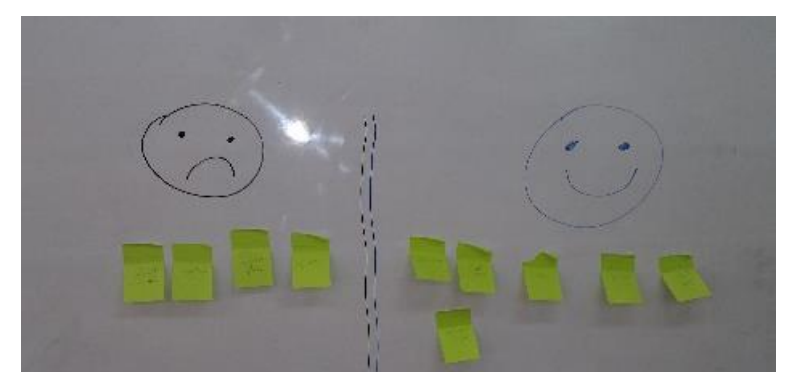

Figure 1: Sticky note activity (Educational problem definition)

appropriate for research with children.

The first activity used photography with instant printing cameras. Children were asked to take a picture that reminds them of education. They then explain the picture and why they chose it. The second activity used sticky notes, where we have two sections on a whiteboard, one with a smiley face and the other with a sad face. Children were asked to name factors that make education either satisfying or dissatisfying.

Children were between the ages of 8 and 15 of mixed gender. Each activity would host around 
seven children with a maximum age difference of two years. We found that inviting groups of friends increase productivity and encourage participation.

Both activities helped us understanding children's perception of education, their educational needs which were strongly linked to literacy in this case study, and the topics that gets them engaged or disaffected in education. Conducting these activities helped to create a positive relationship with the children and made us ready to start the design stage with a clear understanding of the goals.

\subsection{Co-Design}

This stage aims to design solutions in response to identified educational challenges and needs. Design work starts with children envisaging solutions and producing requirements. Children are then asked to create both the pedagogical and technological aspects of the design, including contextual elements. The children's designs are then brought to adult design workshops where they are matched with the available resources such as locations, time, human resources, equipment, and funding to insure applicability and sustainability. This produces designs where the requirements are derived from children to ensure that it is engaging and suitable for them, and at the same time is applicable and sustainable to the context in which it will be used.

In the case study, the activities with children consisted of co-design workshops that involved drawing, LEGO, and modelling. We followed a similar sampling approach to the previous activities but with only three to four children at a time. Inspired by Fisher, Yefimova, and Yafi (2016), children were asked to create a "Magical Learning Machine". They were guided by questions to structure the activity, such as: What and will the machine teach you? Where would it be placed? Will you use it alone or with friends? How will teaching happen? We provided them with a diverse range of printed drawings of hard-to-draw technology equipment so

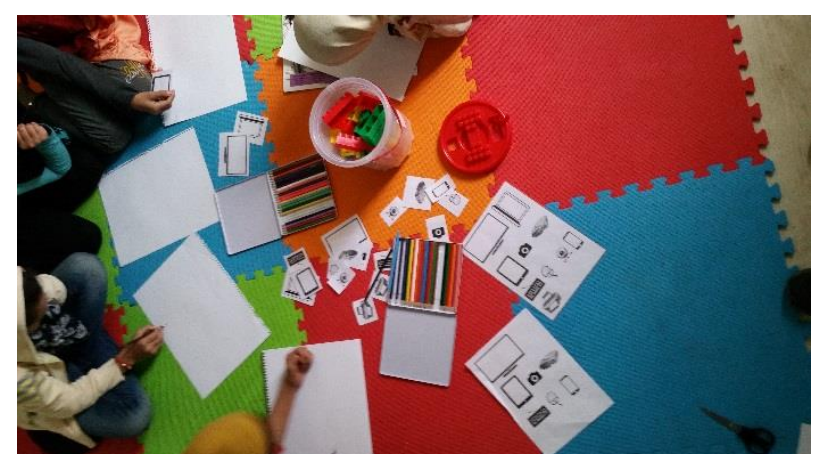

Figure 2: Children's co-design workshop they could include them in their design if they want.

Some children designed self-learning systems used by the child as they wanted autonomy in learning, they justified this by explaining that with self-learning they can learn at their own pace with no influence or interruption. Whereas others designed group learning systems operated by a teacher such as a projector linked to a laptop that shows pictures and videos on the screen to add more visual aids and overcome the language barrier.

The resulted designs, educational needs, and challenges from previous stages, were presented to the adult participants. We worked with them to prioritise the designs by mapping them with the available resources. In this case, due to the unavailability of teachers, the chosen outcome was a digital self-learning space at the camp using an available caravan and a set of tablets that were donated earlier but were not in use. The content was chosen to match the designs of the children to insure usability, and was assessed against the identified conflict sensitive topics such as violence and the aspects of engagement, motivation, and gamification literature. Evaluation sessions were conducted with children throughout the pilot and

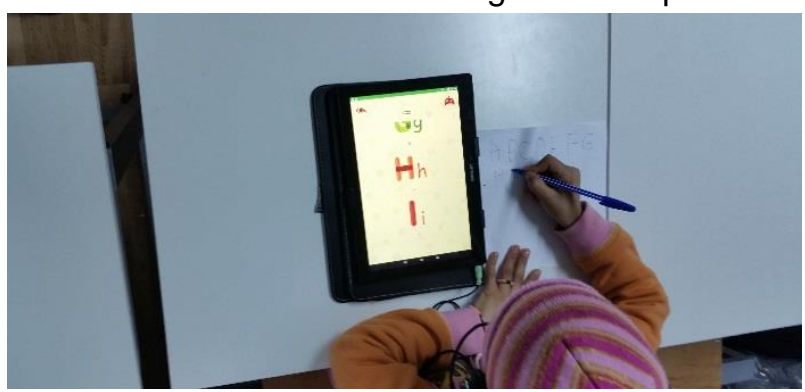

Figure 3: Child practising literacy at the digital learning space implementation phases to insure that the content and final design matches their expectations.

\section{CONCLUSION}

We described a process of designing educational technology solutions for the emergency education of war-affected displaced children based on interdisciplinary research. We have explored how one of the case studies was conducted. The solution devised from this has been implemented and is now a focus for evaluation. Regular assessment sessions and group interviews were conducted with all participant groups to understand their views on the process. The analysis of data from the case studies is ongoing. To date, the key findings are the importance of spending time understanding the conflict, the community, developing appropriate problem definitions, and building trust and relationships with the community. The process enables designers to be inclusive of different groups and to understand the knowledge they can provide and how they can provide it.

Acknowledgments: This research was funded by the Leverhulme Trust under the Open World Learning doctoral scholarship programme at The Open University. In addition, the research team 
would like to thank the "I Am You" NGO and all the participants who facilitated this case study at Ritsona refugee camp in Greece.

\section{REFERENCES}

Betancourt, T. S. (2005) 'Working Paper \#21 Stressors, Supports, and the Social Ecology of Displacement: Psychosocial Dimensions of an Emergency Education Program for Chechen Adolescents Displaced in Ingushetia, Russia', Culture, medicine and psychiatry, 3(29), pp. 309340. Available at:

https://dspace.mit.edu/bitstream/handle/1721.1/976

12/21_stressors.pdf?sequence $=1$ (Accessed: 30 April 2017).

Cohen, L., Manion, L. and Morrison, K. (2013) 'Research Methods in Education, Sixth Edition'. Available at:

https://dspace.utamu.ac.ug/bitstream/123456789/1 82/1/Research-Methods-in-Education-sixthedition.pdf (Accessed: 15 August 2017).

Druin, A. (2002) 'The role of children in the design of new technology', Behaviour \& Information Technology, 21(1), pp. 1-25. doi: 10.1080/01449290110108659.

Druin, A. (2003) 'What Children Can Teach Us: Developing Digital Libraries for Children with Children'. Available at:

https://pdfs.semanticscholar.org/fb0e/4ac039efded e7ef84b1dda3349687188b029.pdf (Accessed: 25 July 2017).

Dryden-Peterson, S. (2011) Refugee Education A Global Review. Available at:

http://www.unhcr.org/4fe317589.pdf (Accessed: 30 May 2017).

Fisher, K. E., Yefimova, K. and Yafi, E. (2016) ““ Future 's Butterflies:" Co-Designing ICT Wayfaring Technology with Refugee Syrian Youth', ACM SIGCHI Conference on Interaction Design and Children 2016, pp. 25-36. doi: http://dx.doi.org/10.1145/2930674.2930701.

Kadiyala Madhavi, C. L. B. (2000) 'A Review of Literature on Effectiveness of Use of Information Technology in Education', Journal of Engineering Education, 89(April), pp. 177-189.

Kagawa, F. (2005) 'Emergency education: a critical review of the field', Comparative Education, 41(4), pp. 487-503. doi: 10.1080/03050060500317620.

Muller, M. J. and Druin, A. (2003) 'Participatory design: The third space in $\mathrm{HCl}$ ', Human-Computer Interaction Handbook, 4235, pp. 1051-1068. doi: 10.1145/153571.255960.
Niemiec, C. P. and Ryan, R. M. (2009) 'Autonomy, competence, and relatedness in the classroom: Applying self-determination theory to educational practice', Theory and Research in Education, 7(2), pp. 133-144. doi: 10.1177/1477878509104318.

Pigozzi, M. J. (1999) 'Education in emergencies and for reconstruction: a developmental approach', UNICEF working paper series, p. 21 p. Available at: http://www.reliefweb.int/rw/lib.nsf/db900sid/LGEL5G8FMF/\$file/unicef-education-1999.pdf CN - PIG 74 (Accessed: 30 April 2017).

Scaife, M. and Rogers, Y. (1999) 'Kids as informants: telling us what we didn't know or confirming what we knew already?', in The Design of Children's Technology. Morgan Kaufmann, pp. 1-26. doi: 10.1145/258549.258789.

Sinclair, M. (2002) Planning education in and after emergencies, International Journal of Educational Development. doi: 10.1016/j.jjedudev.2003.08.003.

Skinner, E. and Pitzer, J. (2012) 'Developmental Dynamics of Student Engagement, Coping, and Everyday Resilience', in Handbook of Research on Student Engagement, pp. 515-539. doi: 10.1007/978-1-4614-2018-7.

Spitzer, D. R. (1996) 'Motivation: The Neglected Factor in Instructional Design', Italian Journal of Educational Technology, 4(3), pp. 38-38.

Talbot, C. and Muigai, K. (1998) 'Environmental education for refugees: guidelines, implementation and lessons learned.', Education as a Humanitarian Response, pp. 223-247.

Tawil, S. and Harley, A. (2004) 'Education and Identity-based Conflict: Assessing curriculum policy for social and civic reconstruction', Education, Conflict and Social Cohesion, pp. 3-33. Available at:

http://www.ibe.unesco.org/fileadmin/user_upload/ar chive/conflict/educ_ind_01.pdf (Accessed: 31 May 2017).

UNHCR (2016a) GLOBAL TRENDS: FORCED DISPLACEMENT IN 2016. Available at: http://www.unhcr.org/5943e8a34.pdf (Accessed: 14 May 2018).

UNHCR (2016b) 'Missing out: Refugee education in crisis', p. 48. 